\title{
Decreased histone deacetylase 4 is associated with human osteoarthritis cartilage degeneration by releasing histone deacetylase 4 inhibition of runt-related transcription factor-2 and increasing osteoarthritis-related genes: a novel mechanism of human osteoarthritis cartilage degeneration
}

Kun Cao ${ }^{1}$, Lei Wei ${ }^{1,2^{*}+}$, Zhiqiang Zhang ${ }^{1}$, Li Guo ${ }^{1}$, Congming Zhang ${ }^{1}$, Yongping Li ${ }^{1}$, Changqi Sun ${ }^{2}$, Xiaojuan Sun ${ }^{2}$, Shaowei Wang ${ }^{1}$, Pengcui $\mathrm{Li}^{1}$ and Xiaochun Wei ${ }^{1 *+}$

\begin{abstract}
Introduction: To investigate if decreased histone deacetylase 4 (HDAC4) is associated with human osteoarthritis $(\mathrm{OA})$ cartilage degeneration by releasing HDAC4 inhibition of runt-related transcription factor-2 (Runx2) resulting in increase of OA cartilage degeneration-related genes.

Methods: The mRNA and protein levels of HDAC4, Runx2, matrix metalloproteinase (MMP)-13, Indian hedgehog (Ihh) and type $X$ collagen were detected by performing real-time PCR (RT-PCR), western blotting and immunohistochemistry on specimens from human $\mathrm{OA}$ and normal cartilage. To further explore the mechanism of regulation of Runx2 and OA-related genes by HDAC4, changes in these OA-related genes were further quantified by RT-PCR after overexpression of HDAC4 and knockdown of HDAC4 by siRNA. Runx2 and MMP-13 promoter activities were measured by dual luciferase assays.
\end{abstract}

Results: The levels of HDAC4 in the cartilage from OA patients and healthy 40- to 60-year-old donors were decreased to $31 \%$ and $65 \%$ compared with specimens from 20 - to 40 -year-old healthy donors, respectively $(P<0.05)$. Decreased HDAC4 was associated with increased Runx2 and other OA-related genes in human OA cartilage, specifically: MMP-13, Ihh and type $X$ collagen. Exogenous HDAC4 decreased the mRNA levels of Runx2, MMP1, MMP3, MMP-13, type X collagen, Ihh, ADAMTS-4 and -5, and increased the mRNA of type II collagen. In addition, the data also shows that overexpression of HDAC4 not only decreased the expression of interleukin (IL)-1 $\beta$, Cox2 and iNos and increased the expression of aggrecan, but also partially blocked the effect of IL-1 $\beta$ on expression of catabolic events in human OA chondrocytes. HDAC4 also inhibited Runx2 promoter activity and MMP13 promotor activity in a dose-dependent manner. In contrast, inhibition of HDAC4 by TSA drug had an opposite effect.

Conclusions: Our study is the first to demonstrate that decreased HDAC4 contributes, at least in part, to the pathogenesis of OA cartilage degeneration. Thus, HDAC4 may have chondroprotective properties by inhibiting Runx2 and OA-related genes.

\footnotetext{
* Correspondence: lei_wei@brown.edu; weixiaochun11@126.com

${ }^{\dagger}$ Equal contributors

'Department of Orthopaedics, Second Hospital of Shanxi Medical University, Shanxi Key Laboratory of Bone and Soft Tissue Injury Repair, Taiyuan, China ${ }^{2}$ Department of Orthopaedics and Department of Surgery, Warren Alpert Medical School of Brown University/Rhode Island Hospital (RIH), Providence, Rhode Island, USA
} 


\section{Introduction}

Osteoarthritis (OA) is the most prevalent joint disease, and is characterized by destruction and loss of articular cartilage, leading to chronic pain and dysfunction. Studies have demonstrated that aging, genetics and mechanical factors are associated with the development of OA. However, the molecular mechanisms involved in the pathogenesis and progression of the disease are not completely understood.

Recent studies have demonstrated that changes observed in the behavior of chondrocytes in OA cartilage resemble those undergone by chondrocytes undergoing differentiation during embryogenesis [1-6]. Hypertrophic chondrocytes express matrix metalloproteases (MMPs) and type $\mathrm{X}$ collagen, which cause cartilage degradation as part of the endochondral ossification process of growth plate development [7-10]. Chondrocytes residing at the ends of the long bones maintain a stable phenotype and are resistant to the hypertrophic state [11]. However, during OA, chondrocytes lose the stable phenotype and enter hypertrophic differentiation, as demonstrated by increased expression of type X collagen, Ihh, and MMP-13. Cartilage degeneration in $\mathrm{OA}$ is therefore similar to cartilage degradation in the growth plate $[12,13]$.

Histone deacetylase 4 (HDAC4) is a member of the class IIa histone deacetylase family. Recent studies show that HADC4 plays an important role in skeletal formation. In the growth plate, HDAC4 prevents chondrocyte hypertrophy by inhibiting the activity of runt-related transcription factor-2 (Runx2), a transcription factor essential for chondrocyte hypertrophy during endochondral bone formation. The HDAC4-null mutant mice display chondrocyte hypertrophy, increased MMP-13, and premature ossification of developing bones due to constitutive expression of Runx2 [14,15]. HDAC4 inhibits MMP-13 through binding to the Runx2 promoter region [15]. Thus, HDAC4 is considered as a central regulator of chondrocyte hypertrophy and skeletogenesis during the growth plate development. However, the role of HDAC4 in OA chondrocytes is unknown. An increase in Runx2 has been reported in OA cartilage and is associated with increases in MMP-13 and type X collagen $[16,17]$. Moreover, decreased HDAC4 mRNA has been reported in cartilage from OA patients when compared with cartilage from healthy donors [18].

Thus, we put forward the hypothesis that decreased HDAC4 may be associated with human OA cartilage degeneration by releasing HDAC4 inhibition of Runx2 and resulting in increased type X collagen, Ihh, and MMP-13. To test the hypothesis, we first detected and compared the expression of HDAC4, Runx2, and OA-related genes between human $\mathrm{OA}$ and normal cartilage. Then we performed a similar comparison after manipulating the expression of HDAC4 in human OA chondrocytes.

\section{Methods}

\section{Cartilage specimens}

Articular cartilage samples were obtained from patients with $\mathrm{OA}$ at the time of knee replacement surgery $(\mathrm{N}=$ 33, 21 females, 12 males, average age $66.58 \pm 7.12$ year (mean \pm standard deviation (SD)), range 56 to 81 years). Normal samples of articular cartilage were obtained from patients undergoing amputation because of trauma $(\mathrm{N}=12$, 2 females, 10 males, average age $39.25 \pm 13.62$, range 21 to 60 ). The normal samples were divided into two groups based on age: 1 ) a 20- to 40-year-old group $(\mathrm{N}=6,2$ females, 4 males, average age $28.33 \pm 7.84$, range 20 to 37$)$; and 2) a 40 - to 60 -year-old group $(\mathrm{N}=6,6$ males, average age $50.17 \pm 7.78$, range 41 to 60 ). Patients with rheumatoid arthritis and other autoimmune diseases, as well as chondrodysplasias and posttraumatic OA, were excluded from this study. Cartilage specimens from patients with OA were obtained from the more affected compartment (usually medial tibial plateaus). Normal cartilage samples from patients undergoing amputation were also obtained from medial tibial plateaus. The absence of cartilage degeneration in the normal cartilage samples was confirmed using Safranin-O staining. The study was approved by the Institutional Review Board at the Second Hospital of Shanxi Medical University, and informed consent was obtained from each tissue donor.

\section{Histology}

Articular cartilages were fixed in $10 \%$ formalin for three days. The specimens were decalcified in EDTA solution, and embedded in a single block of Paraplast X-tra (Thermo Fisher Scientific, Hampton, NH, USA). Serial $6-\mu$ m-thick sections were cut by a rotary microtome (Leica RM2125, Leica Microsystems Ltd, Shanghai, China). Safranin-O staining was performed and cartilage damage was quantified using the modified Mankin grading system [19].

\section{Chondrocyte isolation and primary culture}

Chondrocytes were isolated as previously described [20]. Articular cartilage was dissected with a scalpel and subjected to digestion with $2 \mathrm{mg} / \mathrm{ml}$ proteinase (SigmaAldrich, St Louis, MO, USA) in Hank's balanced salt solution (HBSS) (Invitrogen, Carlsbad, CA, USA) for $30 \mathrm{~min}$ at $37^{\circ} \mathrm{C}$ with shaking. After digestion and removal of the supernatant, the cartilage pieces were washed with Dulbecco's modified Eagle's medium/Ham F-12 (DMEM/ F-12) (Gibco BRL, Paisley, UK), and digested with $1 \mathrm{mg} /$ $\mathrm{ml}$ Type IA collagenase (Sigma-Aldrich, St Louis, MO, USA) for 6 to $8 \mathrm{~h}$ at $37^{\circ} \mathrm{C}$ with shaking. Enzymatic digestion was stopped by adding DMEM/F-12 containing $10 \%$ fetal bovine serum (FBS) (Invitrogen, Carlsbad, CA, USA). Isolated cells were filtered through a $100-\mu \mathrm{m}$ mesh to remove undigested matrix and the cells were washed three times with DMEM/F-12. Isolated chondrocytes 
from individual specimens were separately cultured with DMEM/F-12 plus $10 \% \mathrm{FBS}$ at $37^{\circ} \mathrm{C}$ under a humidified $5 \% \mathrm{CO} 2$ atmosphere until reaching confluence. Chondrocyte phenotype were confirmed by immunocytochemistry using anti-type-I and anti-type-II collagen monoclonal antibodies (SC-25974, SC-7764, Santa Cruz Biotechnology, Santa Cruz, CA, USA). These cells are negative for type I staining and positive for type II collagen.

\section{DNA constructs and transfections}

HDAC4-flag and HDAC4-GFP were a generous gift from Dr A. R. Means [21]; pGL3-Runx2-LUC (0.6 kb of rat RUNX2 promoter) was provided by Dr. G. S. Stein [22]. MMP-13 promoter was a gift provided by Im HJ and Alliston $\mathrm{T}$ [23]. The cells were trypsinized and placed in six-well culture plates (Becton Dickinson Labware, Franklin Lakes, NJ, USA) at a density of $1 \times$ $10^{6}$ cells/plate. The next day, the first passage cells were transfected with HDAC4 small interfering RNA (siRNA) (100 nM), scrambled siRNA control (100 nM) (Cell Signaling Technology, Danvers, MA, USA), or HDAC4flag or control vector $(2.5 \mu \mathrm{g})$ using the GeneMute siRNA transfection reagent (SignaGen Laboratories, Gaithersburg, MD, USA). The cells were harvested for real-time PCR or western blot analysis respectively $48 \mathrm{~h}$ or $72 \mathrm{~h}$ after transfection. To investigate how decreased HDAC4 in articular chondrocytes affects expression of catabolic events in these cells after the treatment with interleukin (IL)-1 beta, the OA chondrocytes were transfected with HDAC4 or control constructs for $48 \mathrm{~h}$, then the cells were stimulated with or without IL-1 beta $(5 \mathrm{ng} / \mathrm{ul})$ for $24 \mathrm{~h}$. After IL- 1 beta treatment, these cells were harvested for real-time PCR. In order to confirm the transfection efficiency, HDAC4GFP or siRNA control (fluorescent conjugate) (Cell Signaling Technology, Danvers, MA, USA) were transfected to OA chondrocytes. Forty-eight hours after transfection, live green fluorescent protein (GFP)-positive cells were counted under fluorescence microscopy. Each measurement was made in triplicate.

\section{Immunohistochemistry staining}

Immunohistochemistry was performed to detect the distribution of Runx2、Ihh type X collagen and MMP-13 in the cartilage specimens. Six- $\mu$ m paraffin sections were deparaffinized and treated with $3 \%$ hydrogen peroxide (Sigma-Aldrich, St Louis, MO, USA) to block intrinsic peroxidase in methanol (Sigma-Aldrich, St Louis, MO, USA) for $30 \mathrm{~min}$. The sections were digested by $5 \mathrm{mg}$ / $\mathrm{ml}$ hyaluronic acid (HA) in phosphate-buffered saline (PBS) (Sigma-Aldrich, St Louis, MO, USA) for $20 \mathrm{~min}$. Normal sheep serum (LI-COR Biosciences, Lincoln, NE, USA) was used to prevent nonspecific binding. The sections were incubated with primary antibodies against human Runx2 (CeMines, Inc, Golden, CO, USA), Ihh,
MMP-13 (Santa Cruz Technology, Santa Cruz, CA, USA) and type X collagen (EMD, Gibbstown, NJ, USA) respectively at $4^{\circ} \mathrm{C}$ overnight. The negative control sections were incubated with IgG isotype control $(2 \mathrm{mg} / \mathrm{ml})$ (R\&D, Minneapolis, MN, USA) in PBS. The second day, the sections were treated sequentially and incubated with biotinylated secondary antibody and streptavidinperoxidase conjugate (Zymed-Invitrogen, Carlsbad, CA, USA), followed by standardized development in 3'3-diaminobenzidine (DAB). The sections were counterstained with hematoxylin (Zymed-Invitrogen, Carlsbad, CA, USA). Photography was performed with a Nikon E800 microscope (Nikon, Melville, NY, USA).

To detect the expression of HDAC4 in cartilage, $6-\mu \mathrm{m}$ sections were analyzed by immunofluorescent staining. The sections were incubated with goat primary antibody (sc-5245 Santa Cruz Technology, Santa Cruz, CA, USA), at $4^{\circ} \mathrm{C}$ overnight. The negative control sections were incubated with isotype control (sc-1196-P, Santa Cruz Technology, Santa Cruz, CA, USA) in PBS. After washing with PBS, affinity-purified tetramethylrhodamine (TRITC)-conjugated donkey anti-goat secondary antibody (1:500) (Jackson ImmunoResearch Laboratories, West Grove, PA, USA) was applied with Hoechst nuclear dye $(0.5 \mathrm{mg} / \mathrm{ml})$ (Pierce, Rockford, IL, USA). The sections were washed and mounted in GEL/MOUNT ${ }^{\text {тм }}$ (Biomeda Corporation, Foster City, CA, USA). Photography was performed with a Nikon E800 microscope (Nikon, Melville, NY, USA).

To confirm that the OA chondrocytes used in this study were not dedifferentiated, we performed immunohistochemistry with type II collagen and type I collagen staining. $1.5 \times 10^{5}$ cells were seeded on the slide, then fixed in $4 \%$ paraformaldehyde for $20 \mathrm{~min}$ at $4^{\circ} \mathrm{C}$ after culture for $48 \mathrm{~h}$. After fixation, cells were permeabilized for $10 \mathrm{~min}$ in $0.3 \%$ Triton X-100 in PBS and incubated in $3 \%$ bovine serum albumin for $1 \mathrm{~h}$. The cells were incubated with primary antibodies against type II collagen and type I collagen overnight at $4^{\circ} \mathrm{C}$ (SC-25974, SC-7764, Santa Cruz Technology, Santa Cruz, CA, USA). The cells were then incubated with a Texas Red-conjugated secondary antibody (SC-2783, Santa Cruz Technology, Santa Cruz, CA, USA) for $30 \mathrm{~min}$ at room temperature, followed by counterstaining with Hoechst 33242 (Pierce, Rockford, IL, USA). Photography was performed with a Nikon E800 microscope (Nikon, Melville, NY, USA).

\section{Western blotting}

Lysis buffer was used to extract total protein from OA cartilage and normal cartilage specimens or from chondrocytes $72 \mathrm{~h}$ after transfection with HDAC4 or HDAC4 siRNA. Protein concentrations were determined with the BCA Protein Assay Kit (Pierce, Rockford, IL, USA). Total protein (40 ug) underwent electrophoresis in 10\% SDS 
PAGE gels under reducing conditions. After electrophoresis, proteins were electroblotted onto a PVDF membrane from the gel by a wet blotting apparatus (Thermo Fisher Scientific, Hampton, NH, USA). The membranes were blocked in Tris-buffered saline (TBS) containing 0.1\% Tween-20 and $5 \%$ dry milk at $4{ }^{\circ} \mathrm{C}$ overnight. The next day, the membranes were incubated for $6 \mathrm{~h}$ at room temperature with primary antibodies against HDAC4, Runx2, Ihh, MMP-13 and Col $x$. The primary antibodies were diluted 1:500 in TBS containing 0.1\% Tween-20 (Sigma-Aldrich, St Louis, MO, USA). Immunoglobulin G (IgG)-conjugated with horseradish peroxidase (HRP) was diluted 1:2000 in TBS containing 0.1\% Tween-20 and used as the secondary antibody. The immunoreactive proteins were detected by chemiluminescence using a commercial enhanced chemiluminescence (ECL) kit. Parallel gels were prepared for Coomassie Blue staining to confirm equal loading of total proteins from cartilage samples. The membranes were stripped and probed again with anti- $\beta$ actin, to estimate the total protein for equal loading. The densities of bands were quantified using ChemiDoc ${ }^{\text {tix }}$ XRS + Software (Bio-Rad, Hercules, CA, USA). The following primary antibodies were used for western blot analysis from Santa Cruz Biotechnology, Santa Cruz, CA, USA. Goat anti-HDAC4 (sc-5245), goat anti-Runx2 (sc-12488), goat anti-collagen 10A1 (sc-323750), goat anti-MMP-13 (sc-31813), goat anti-Ihh (sc-1196), mouse anti- $\beta$-actin (sc-130301), rabbit anti-goat IgG-HRP (sc-2768), mouse anti-goat IgG-HRP (sc-2354).

\section{Real time RT-PCR}

Total RNA was isolated from OA cartilage and normal cartilage or from chondrocytes transfected with HDAC4 or HDAC4 siRNA $48 \mathrm{~h}$ after the transfection, using Trizol reagent (Invitrogen, Carlsbad, CA, USA). The RNA was reverse-transcribed into first-strand complementary cDNA using the iScript ${ }^{\mathrm{m}}$ cDNA synthesis kit (Bio-Rad, Hercules, CA, USA). The quantification of mRNA was determined using the QuantiTect SYBR Green PCR kit (Qiagen, Valencia, CA, USA) according to the manufacturer's protocols. Each reaction was performed in triplicate. HADC4 primers were as follows: forward, 5 '-GAG AGA CTC ACC CTT CCC G-3', and reverse, 5' -CCG GTCTGC ACC AAC CAA G-3'. Runx2 primers were as follows: forward, 5'-GGC AGG CAC AGT CTT CCC-3', and reverse, 5'- GGC CCA GTT CTG AAG CAC C -3'. Ihh primers were as follows: forward, $5^{\prime}$-CAT TGA GAC TTG ACT GGG CAA C-3', and reverse, 5'-AGA GCA GGC TGA GTT GGG AGTCGC-3'. Type II collagen primers were as follows: forward, 5'-TGA GGG CGC GGT AGA GAC CC-3', and reverse, 5' -TGC ACA CAG CTG CCA GCC TC-3'. Type X collagen primers were as follows: forward, 5'-GCA CGC AGA ATC CAT CTG AGA ATA- $3^{\prime}$, and reverse, $5^{\prime}$-GAC CAG GAG TAC CTT
GCT CTC -3 '. MMP-1 primers were as follows: forward, 5'-CCA AAT GGG CTT GAA GCT G-3', and reverse, 5'-GGT ATC CGT GTA GCA CAT TCT GTC-3'. MMP-3 primers were as follows: $5^{\prime}$-TTT CCA GGG ATT GAC TCA AAG A-3', and reverse, 5'-AAG TGC CCA TAT TGT GCC TTC-3'. MMP-13 primers were as follows: forward, 5'-TGC TGCATT CTC CTT CAG GA-3', and reverse, 5'-ATG CAT CCA GGG GTC CTGGC-3'. IL-1 beta primers were as follows: forward, $5^{\prime}$-TCG CCA GTG AAA TGA TGG CTT A-3', and reverse 5'-GTC CAT GGC CAC AAC AAC TGA-3'. iNos primers were as follows: forward, $5^{\prime}$-CCT TAC GAG GCG AAG AAG GAC AG-3', and reverse, 5' -CAG TTT GAG AGA GGA GGC TCC G-3'. Cox2 primers were as follows: forward, 5'-GAG AGATGTATC CTC CCA CAG TCA-3', and reverse, 5'- GAC CAG GCA CCA GAC CAA AG-3'. ADAMTS-4 primers were as follows: forward, 5'-GAC ACG CTG GGT ATG GCT GA-3', and reverse, 5' ${ }^{\prime}$-ACT GAT GCA TGG CTT GGA GTT G-3'. ADAMTS-5 primers were as follows: forward, 5'-TAT GAC AAG TGC GGA GTA TG -3' and reverse, 5' -TTC AGG GCT AAA TAG GCA GT-3'. Aggrecan primers were as follows: forward, 5' - CAT TCA CCA GTG AGG ACC TCG T-3' and forward, 5'-TCA CAC TGC TCA TAG CCT GCT TC-3'. Amplification conditions were as follows: 2 min of preincubation at $50^{\circ} \mathrm{C}, 10 \mathrm{~min}$ at $95^{\circ} \mathrm{C}$ for enzyme activation, and 40 cycles at $95^{\circ} \mathrm{C}$ denaturation for $10 \mathrm{~s}, 55^{\circ} \mathrm{C}$ annealing for $30 \mathrm{~s}$, and $72^{\circ} \mathrm{C}$ extension for $30 \mathrm{~s}$. $18 \mathrm{~S}$ was used as an internal control gene to normalize the mRNA levels. The cycle threshold $(\mathrm{Ct})$ values for $18 \mathrm{~s}$ RNA and that of target genes were measured and calculated by computer software (IQ50, Bio-Rad Laboratories, Hercules, CA, USA). Relative transcription levels were calculated as $x=2^{-\Delta \Delta C T}$, was used for the calculation of fold amplification [24].

\section{Reporter gene assays}

Prehypertrophic chondrocytes were isolated from the cephalic part of the sternum of a 17-day-old embryonic chick as previously described $[25,26]$. Human chondrocyte cell line (C28/12) and chicken chondrocytes were transiently transfected with $0.5 \mu \mathrm{g}$ of Runx 2 or MMP-13 promoter luciferase reporter plasmid using $5 \mu \mathrm{L} /$ well of lipofectamine 2000 (Invitrogen, Carlsbad, CA, USA) in 12 -well plates. Co-transfections with promoters were performed with empty vector or different concentrations of expression constructs of HDAC4 as indicated. In some case, the cells transfected with promoters were treated with different concentrations of HDAC inhibitor trichostatin A (TSA) as indicated. Cells were harvested in $1 \times$ reporter lysis buffer $48 \mathrm{~h}$ after transfection. Luciferase activity of Runx 2 was read in a luminometer using the dual luciferase reporter assay and normalized to Renilla activity (Dual-Luciferase Reporter Assay, Promega, 
Madison, WI, USA). Each measurement was made in triplicate.

\section{Statistical analysis}

Analysis of variance (ANOVA) was used to compare the significant differences among the concentrations of HDAC4 in normal age-matched control and OA cartilage specimens. Two-tailed paired $t$ tests were used to compare statistically significant differences of the protein (MMP-13, Ihh, type X collagen) in OA and control cartilage specimens. Student's $t$ test was used to compare mRNA levels in cartilage or chondrocytes. ANOVA was used to compare significant differences among the effects on promoter activity of Runx2 or MMP13 with different concentrations of HDAC4 constructs or TSA. ANOVA was used to compare significant differences among the effects of HDAC4 on catabolic markers in
IL-beta-treated chondrocytes. $P<0.05$ was considered significant.

\section{Results}

Decrease of HDAC4 protein found by western blotting in human aging and $O A$ cartilage

First, we analyzed expression of HDAC4 in cartilage samples from the knee joints of OA patients and compared it to that in normal cartilage samples from 20 - to 40 -yearolds and 40- to 60-year-olds. Safranin O staining demonstrated degeneration in OA cartilage, and the lack thereof in normal cartilage (Figure 1A). Western blotting analysis showed that expression of HDAC4 were decreased in the 40- to 60-year-olds and further decreased in the OA specimens, compared with the specimens obtained from the 20 - to 40-year-olds (Figure 1B). $\beta$-actin and Coomassie Blue staining confirmed equal loading of total proteins

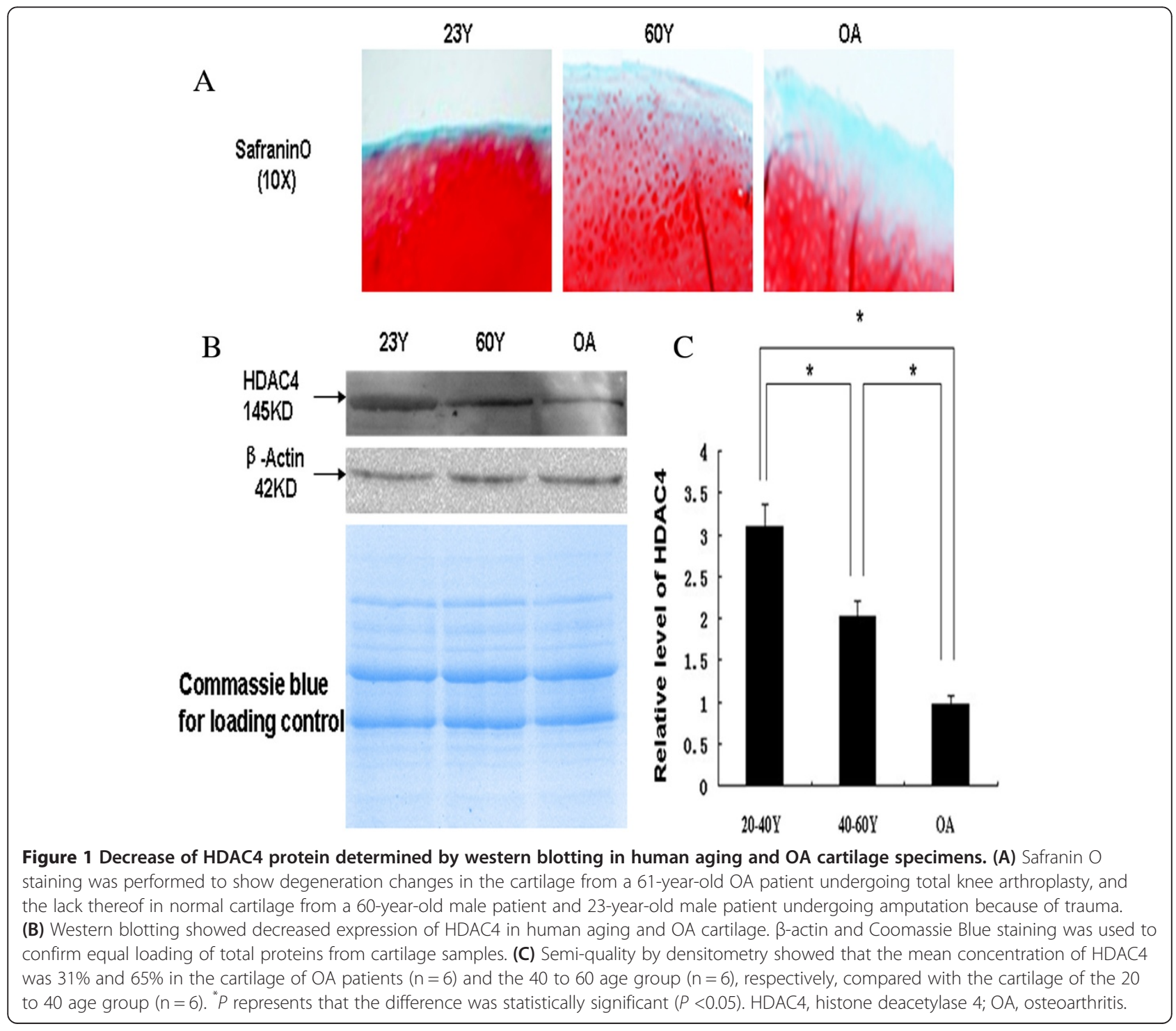


from cartilage samples (Figure 1B). Semi-quantification of western blots showed that, compared with cartilage specimens from the 20- to 40-year-old group, those from the OA and 40- to 60-year-old group had 31\% and $65 \%$ of the concentration of HDAC4, respectively. $(P<0.05)$ (Figure 1C).

\section{Increased expression of RUNX2 was associated with decreased HDAC4 in OA cartilage}

To evaluate the potential relationship between HDAC4 and Runx2 as dysregulated pathways in OA cartilage, we analyzed expression of HDAC4 and Runx2 in cartilage from $\mathrm{OA}$ patients and age-matched normal cartilage specimens from the 40- to 60-year-old group (herein after called 'normal cartilage'). Immunofluorescent staining for HDAC4 was significantly lower in OA cartilage specimens compared with normal cartilage (Figure 2B). HDAC4-positive cells were $17 \%$ of total cells in OA cartilage compared with $90 \%$ in normal cartilage $(P<0.05)$ (Figure 2B). Immunohistochemistry staining showed increased Runx2 in OA cartilage compared with normal cartilage (Figure $2 \mathrm{C}-\mathrm{a}$ ), and real-time PCR and western blotting yielded similar findings (Figure $2 \mathrm{C}-\mathrm{b}, \mathrm{C}-\mathrm{c}, \mathrm{C}-\mathrm{d}$ ). Runx2 gene expression was increased 4.4-fold in $\mathrm{OA}$ cartilage compared with the normal controls. $(P<0.05)$ (Figure 2C-b). Quantification of western blots showed

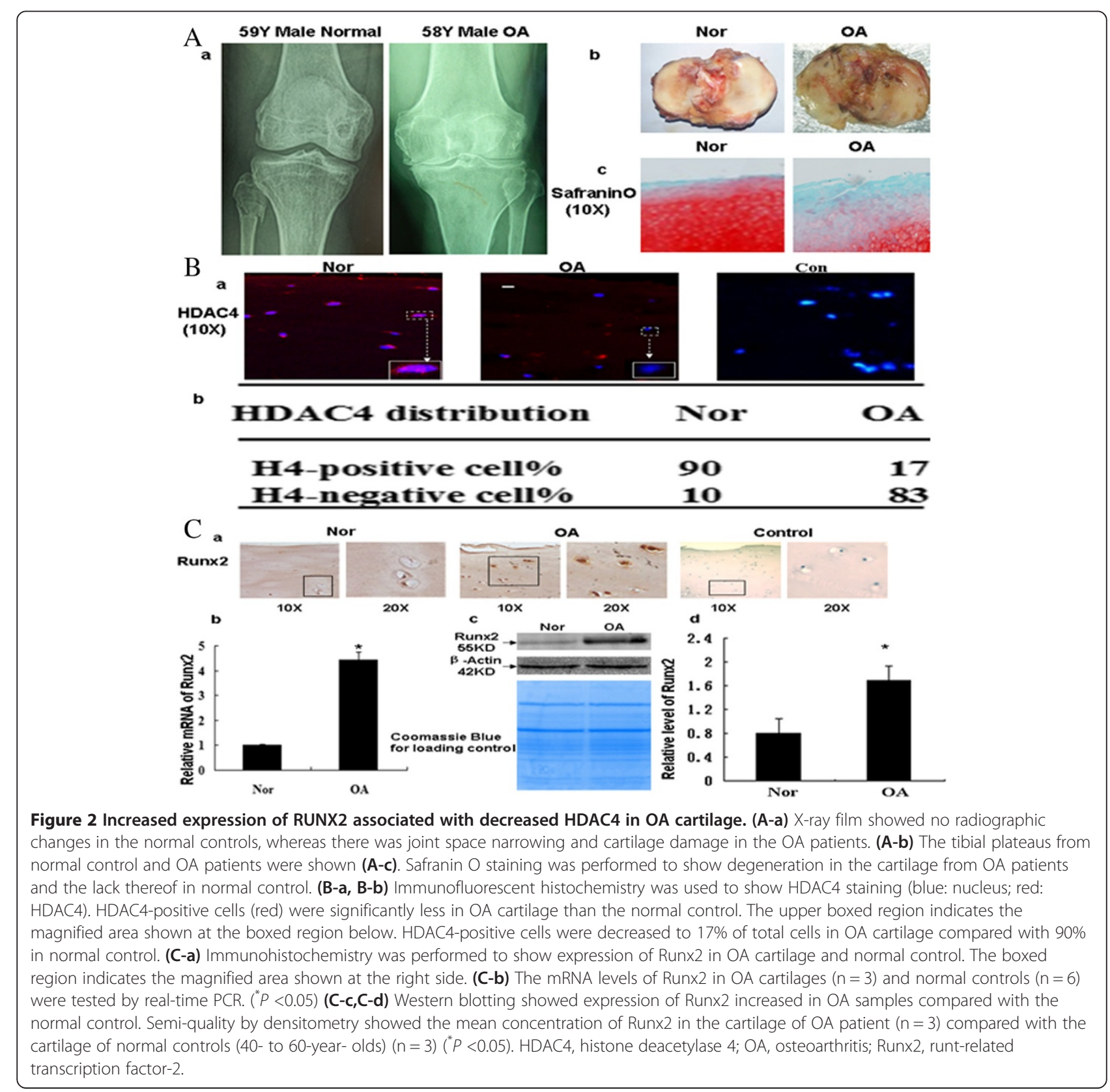


that the concentration of Runx2 in the cartilages of OA patients was increased $212 \%$ compared with the normal cartilages $(P<0.05)$ (Figure $2 \mathrm{C}-\mathrm{d})$.

\section{Increased MMP-13, Ihh and type $\mathrm{X}$ collagen expression in OA cartilage}

Strong staining for MMP-13, Ihh and type X collagen was observed in OA cartilage compared with normal cartilage (Figure 3A). Real-time PCR results indicated that the level of MMP-13, Ihh and type X collagen increased 5.24-, 2.98 - and 3.65-fold, respectively, in OA cartilages compared with normal cartilage $(P<0.05)$ (Figure 3B). Similar findings were made by western blotting. (Figure 3C). Semi-quantification of western blots showed that the concentration of MMP-13, Ihh and type X collagen was increased $278 \%, 257 \%$ and $251 \%$ in cartilage from OA patients compared with those from normal controls, respectively $(P<0.05)$ (Figure $3 C, D)$.
HDAC4 inhibits Runx2 and MMP-13 expression in human OA chondrocytes

Primary chondrocyte phenotype was confirmed by immunocytochemistry, with negative type I staining and positive type II collagen (Figure 4A). To confirm the transfection efficiency, HDAC4-GFP or siRNA control (fluorescent conjugate) were transfected to OA chondrocytes. Our data demonstrated that the average transfection efficiency of GFP-HDAC4 or siRNA control was around $79.1 \%$ (72.6\% to $87.6 \%)$ or $88.2 \%$ (85.4\% to $91.2 \%$ ), respectively (Figure S1 in Additional file 1). To test whether decreased HDAC4 was associated with increased Runx2 in OA cartilages, OA chondrocytes were transfected with different concentrations of HDAC4 $(1 \mu \mathrm{g}$ or $2.5 \mu \mathrm{g})$ or HDAC4 siRNA (100nM) against HDAC4. Real-time PCR demonstrated that HDAC4 inhibited Runx 2 mRNA in a dose-dependent manner. The mRNA levels of Runx2 in OA chondrocytes transfected

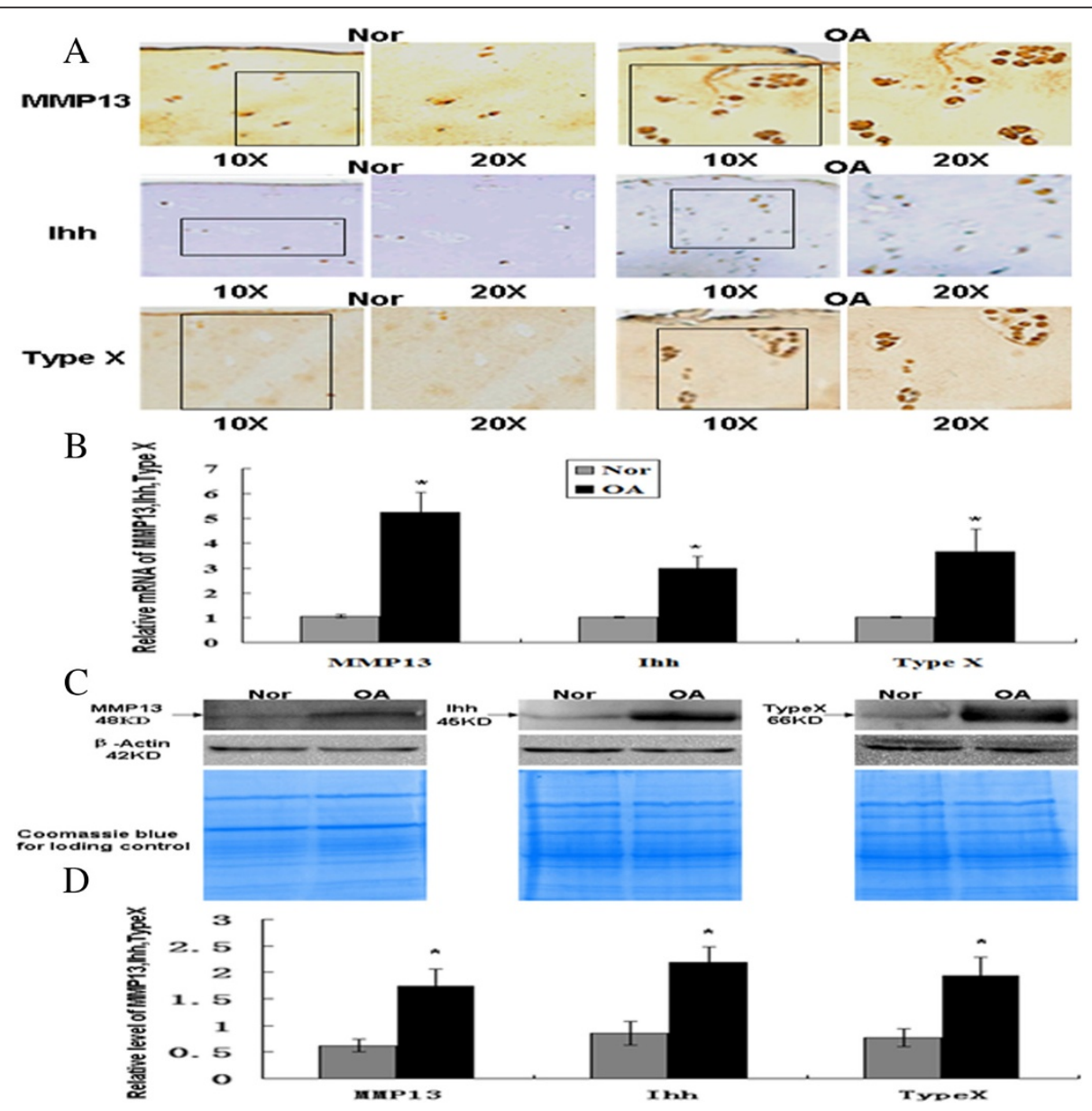

Figure 3 Increased MMP-13, Ihh and type $\mathrm{X}$ collagen expression in OA cartilage. (A) Immunohistochemistry was performed to detect MMP-13, Ihh and type $X$ collagen. The boxed regions indicate the magnified area shown at the right side. (B) Real-time PCR indicates that the level of MMP-13, lhh and type X collagen increased in OA cartilages $(n=3)$ compared with normal cartilage $(n=3)\left({ }^{*} P<0.05\right)$. (C, D) Western blotting showed expression of MMP-13, Ihh and type $X$ collagen increased in OA samples compared with normal controls. Semi-quality by densitometry showed the mean concentration of MMP-13, lhh and type $X$ collagen in the cartilage of OA patients $(n=3)$ compared with the cartilage of normal controls (40- to 60-year-olds) $(n=3)\left({ }^{*} P<0.05\right)$. MMP, matrix metalloprotease; OA, osteoarthritis; SD, standard deviation. 


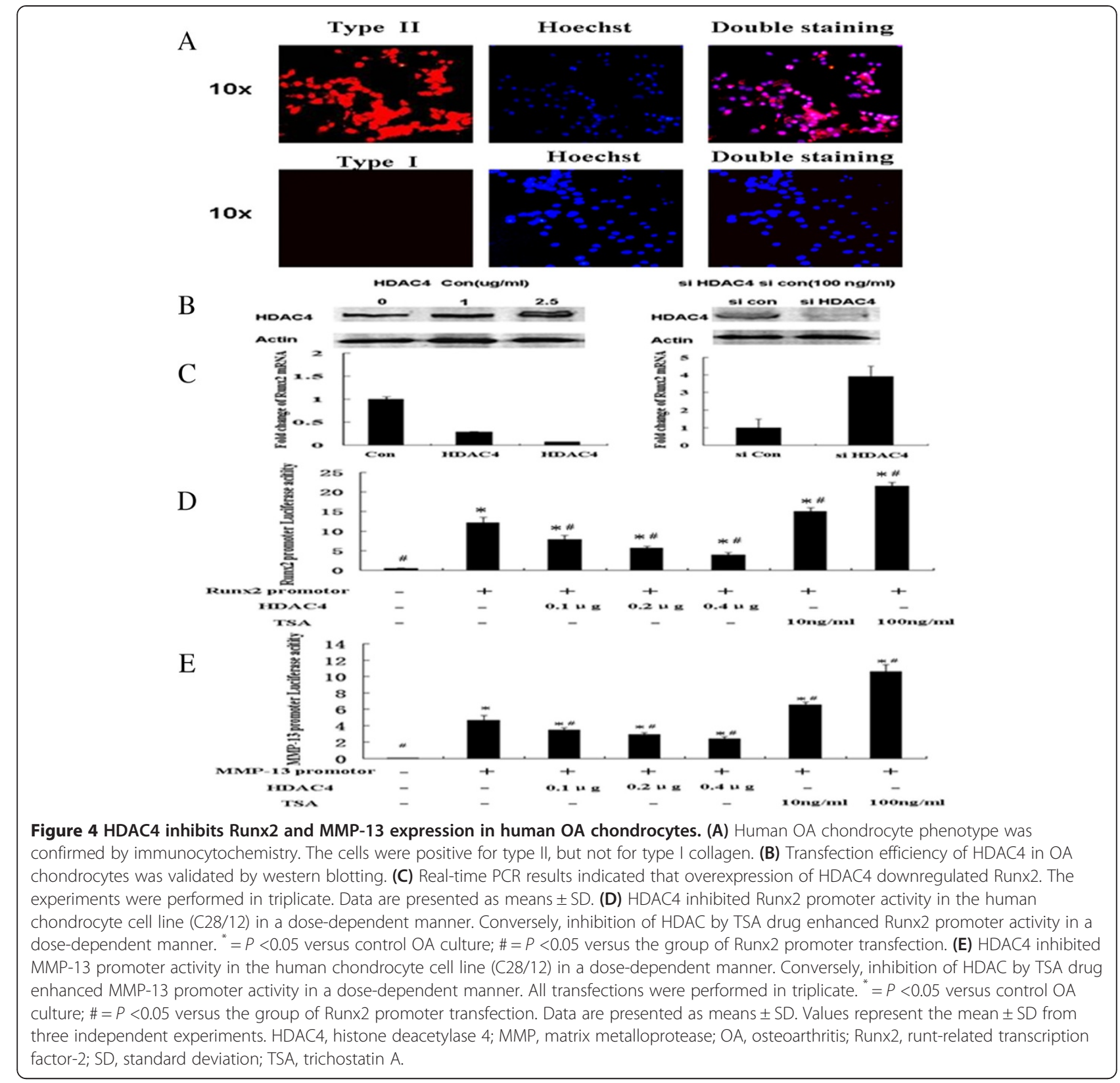

with low and high concentration of HDAC4 decreased to $29 \%$ and $7 \%$ respectively, of those in the control vector (Figure 4C). Increased HDAC4 protein was confirmed by western blotting (Figure 4B). Conversely, increased mRNA of Runx2 was observed in the cells transfected with HDAC4 siRNA. Western blotting showed that HDAC4 siRNA effectively knock down the expression of HDAC4, whereas Runx2 mRNA increased 3.9-fold (Figure 4B, C). To further explore the mechanism of the regulation of Runx 2 and MMP-13 by HDAC4, human chondrocyte cell line (C28/12) and chicken chondrocytes (data not shown) transfected with Runx2 or MMP-13 reporter constructs were treated or co-transfected with different concentrations of TSA (10 ng/ml or $100 \mathrm{ng} / \mathrm{ml})$ or HDAC4 $(0.1 \mu \mathrm{g}, 0.2 \mu \mathrm{g}$ or $0.4 \mu \mathrm{g}$ ) respectively. Dual luciferase assay was performed $48 \mathrm{~h}$ after transfection. Runx2 or MMP-13 promoter activity was repressed in a dose-dependent manner by HDAC4. Conversely, inhibition of HDAC by TSA increases Runx2 or MMP-13 promoter activity in a dose-dependent manner $(P<0.05)$ (Figure 4D, E).

\section{HDAC4 downregulates OA-related gene expressions in human $O A$ chondrocytes}

Our data showed that overexpression of HDAC4 decreased the mRNA levels of Ihh, type X collagen, MMP1, MMP3, MMP-13, IL-1 beta, Cox2, iNos, ADAMTS-4 and -5 in 
OA chondrocytes to $11 \%, 23 \%, 33.8 \%, 23.7 \%, 33 \%, 60 \%$, $57.7 \%, 54.7 \%, 45.1 \%$ and $48.9 \%$, respectively, of those in controls (Figure 5A). In addition, the mRNA levels of type II collagen and aggrecan significantly increased 4.7- and 9.0-fold after HDAC4 transfection (Figure 5A) $(P<0.05)$. In contrast, knockdown HDAC4 by HDAC4 siRNA showed an opposite effect (Figure 5B) $(P<0.05)$. The transfection efficiencies of HDAC4 and HDAC4 siRNA were validated by real-time PCR (Figure 5A, B).

HDAC4 partially blocks the effect of IL-1 beta on expression of catabolic events in human OA chondrocytes

Real-time PCR showed that IL-1 beta significantly increased the mRNA levels of MMP-1, MMP3, MMP-13, iNOS, Cox2, ADAMTS- 4 and -5 in the OA chondrocytes, respectively compared to those untreated controls. In addition, the mRNA levels of aggrecan, and type II collagen significantly decreased after stimulation with IL-1 beta. Overexpression of HDAC4 partly blocked the upregulation of MMP-1, MMP3, MMP-13, iNOS, Cox2, ADAMTS- 4 , and -5 induced by IL- 1 beta. In the meanwhile, the mRNA levels of aggrecan, and type II collagen significantly increased in those cells transfected by HDAC4 (Figure 6) $(P<0.05)$.

\section{Discussion}

Studies have demonstrated that the class II HDACs are involved in cellular development and differentiation, and expressed in a tissue-specific pattern. HDAC4 is expressed in brain, muscle and cartilage $[27,28]$. In the growth plate, HDAC4 is a negative regulator of chondrocyte hypertrophy by binding to and inhibiting Runx2 - a critical transcriptional factor for chondrocyte hypertrophy $[14,26]$. However, the role of HDAC4 during OA cartilage degeneration
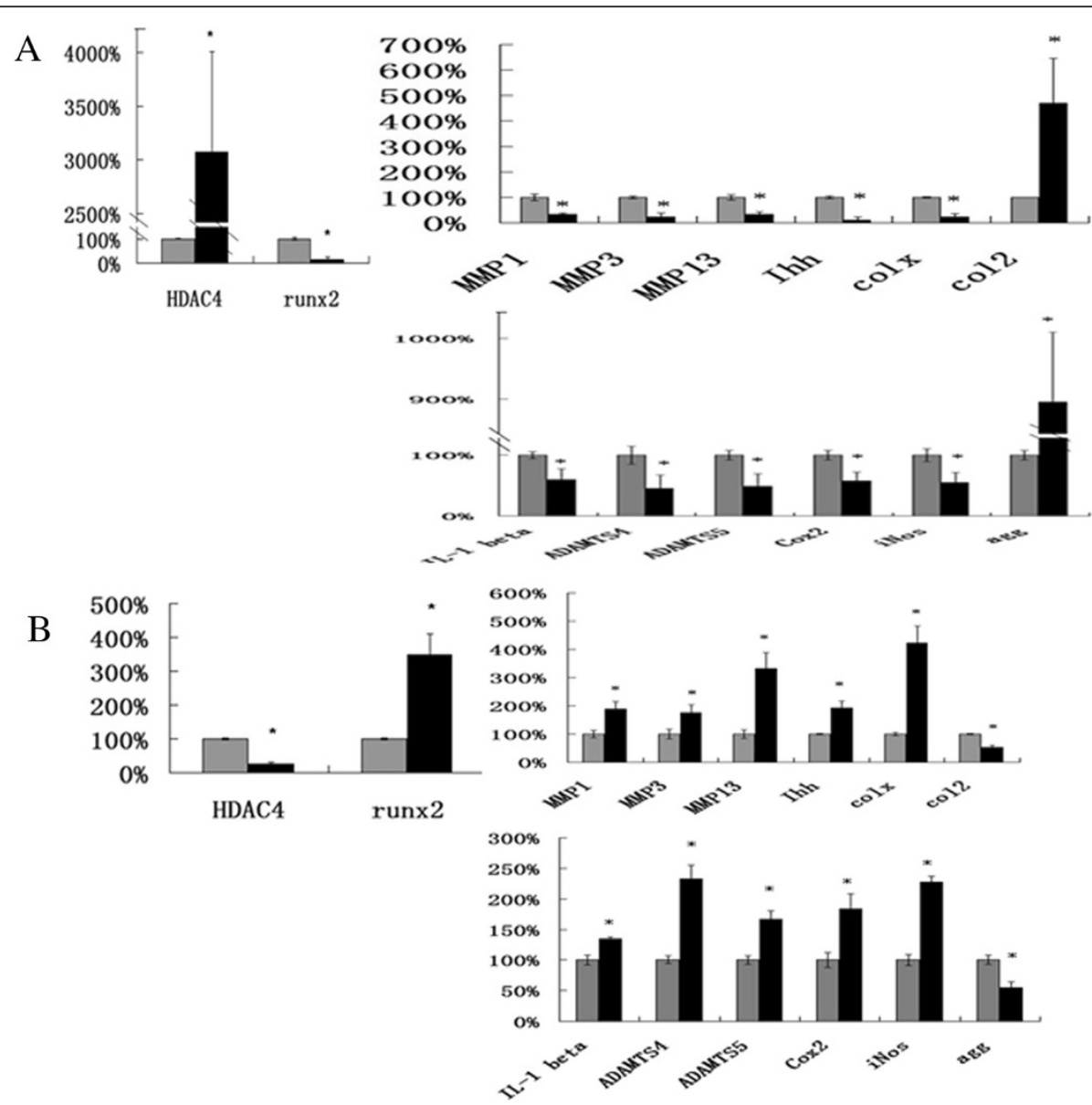

Figure 5 HDAC4 downregulates OA-related gene expressions in human OA chondrocytes. Human OA chondrocytes were transfected with HDAC4-flag or HDAC4 siRNA. All transfections were performed in triplicate. Data are presented as means \pm SD. ${ }^{*} P<0.05$. (A) The mRNA of HDAC4, Runx2, MMP1, MMP3, MMP-13, type X collagen, Ihh, type II collagen, IL-1 beta, Cox2, iNos, ADAMTS-4 and -5 was tested by real-time PCR after HDAC4 or control vector transfection. (B)The mRNA of HDAC4, Runx2, MMP1, MMP3, MMP-13, type X collagen, Ihh, type II collagen, IL-1 beta, Cox2, iNos, ADAMTS-4 and -5 was tested by real-time PCR after HDAC4 siRNA or scrambled siRNA control transfection. HDAC4, histone deacetylase 4; MMP, matrix metalloprotease; OA, osteoarthritis; Runx2, runt-related transcription factor-2; SD, standard deviation; siRNA, small interfering RNA; TSA, trichostatin A. 

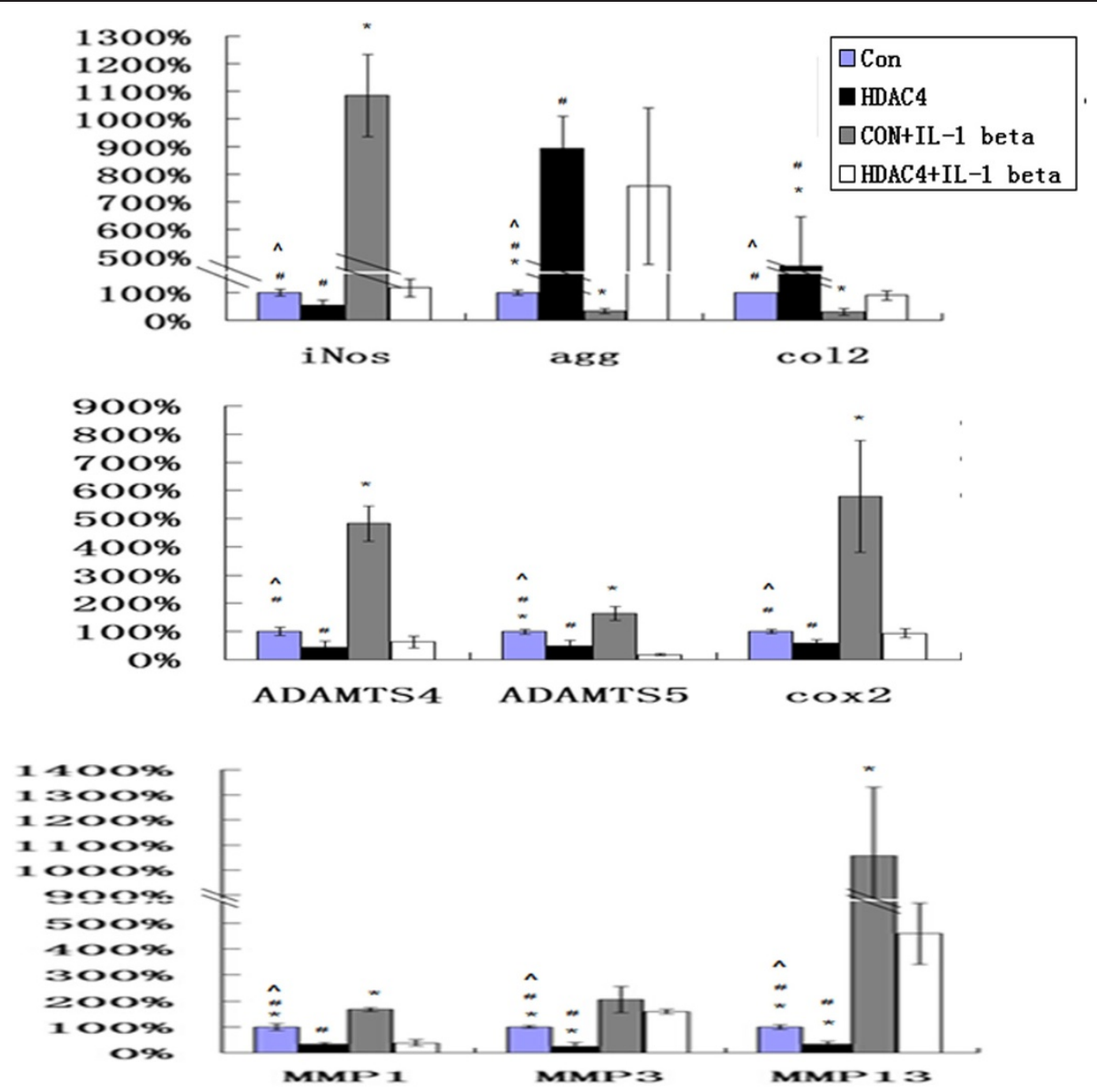

Figure 6 HDAC4 partially blocks the effect of IL-1 beta on expression of catabolic events in human OA chondrocytes. Human OA chondrocytes were transfected with HDAC4 or control constructs for $48 \mathrm{~h}$, then the cells were stimulated with or without IL-1 beta (5 ng/ ul) for $24 \mathrm{~h}$. All transfections were performed in triplicate. ${ }^{*}=P<0.05$ versus the group of HDAC4 + IL-1 beta, \# $=P<0.05$ versus the group of control vercor, $\wedge=P<0.05$ versus the group of control vector $+I L-1$ beta. Real-time PCR showed that IL-1 beta significantly increased the mRNA levels of MMP-1, MMP3, MMP-13, iNOS, Cox2, ADAMTS-4 and -5 in the OA chondrocytes, respectively compared to those untreated controls. In addition, the mRNA levels of aggrecan, and type II collagen significantly decreased after stimulation with IL-1 beta $(P<0.05)$. Overexpression of HDAC4 partly blocked the upregulation of MMP-1, MMP3, MMP-13, iNOS, Cox2, ADAMTS-4, and -5 induced by IL-1 beta. In the meanwhile, the mRNA levels of aggrecan, and type II collagen significantly increased in those cells transfected by HDAC4 $(P<0.05)(n=3)$. HDAC4, histone deacetylase 4; IL, interleukin; MMP, matrix metalloprotease; OA, osteoarthritis; Runx2, runt-related transcription factor-2.

is unknown. In this study, for the first time, we found that expression of HDAC4 protein decreases with aging in human cartilage, and furthermore in human OA cartilage (Figure 1).

In addition to decreased HDAC4, we found that expression of Runx2, Ihh, MMP-13 and type X collagen is increased in OA cartilage with respect to normal controls (Figures 2 and 3). This result is also consistent with previous reports [20,29-32]. Runx2 is a critical regulator for chondrocyte hypertrophy and contributes to the progression of OA. Previous studies have reported that Runx2 is activated in OA cartilage and responsible for the increase of MMP-13 and type X collagen in a mouse OA model and in human OA chondrocytes $[8,10,17,33]$. Type $\mathrm{X}$ collagen and MMP-13 are markers for chondrocyte hypertrophy, and MMP-13 is a primary player in OA cartilage damage [32,34-36]. In an in vivo study, the heterozygous Runx2-deficient mice showed decreased cartilage damage and reduced type X collagen and MMP- 13 expression compared with wild-type mice [10]. These findings suggest that increased Runx 2 in OA cartilage may trigger the expression of a series of genes related to OA cartilage damage. However, it is unknown how Runx2 is regulated in OA cartilage. Taken together with the dysregulation of the genes in OA cartilage shown in our study (Figures 2 and 3), it is likely that decreased HDAC4 is at least partially responsible for the increase of Runx2 and OA-related genes in OA cartilage. We hypothesize that decreased HDAC4 in OA cartilage will release the HDAC4 inhibition of Runx2, thus resulting 
in the increase of Runx2 activity. Thus, upregulation of Runx2 activates a series of OA-related genes and results in cartilage degeneration.

To test whether decreased HDAC4 makes a contribution to increased Runx2 and other OA-related genes in human OA cartilage, OA chondrocytes were transfected with either HDAC4 expression construct or siRNA against HDAC4. Our data showed that either the mRNA level of Runx2 were repressed after overexpression of HDAC4, and the opposite effect was observed when HDAC4 was knocked down by siRNA (Figures 4 and 5). Moreover, we also demonstrated that HDAC4 inhibited Runx2 and MMP-13 promoter activities in a dosedependent manner (Figure 4D, E). In contrast, inhibition of HDAC4 by TSA increased Runx 2 and MMP-13 promoter activities. HDAC4 and Runx2 are known as important regulators of chondrocyte hypertrophy during growth plate development. It is reported that HDAC4 repressed chondrocyte hypertrophy by binding to and inhibiting Runx 2 transcriptional activity in the chondrocytes of an embryonic chick [26]. HDAC4-null mice display ectopic and premature mineralization of endochondral bones, mimicking the Runx2 gain of function mice [14]. Consistent with this finding, Runx2, Ihh and MMP-13 are greatly increased in HDAC4-/- mice [14,15]. It is also reported that HDAC4 inhibited MMP-13 through Runx 2 and HDAC4 and 5 were required for transforming growth factor- $\beta$-mediated repression on Runx2 function to inhibit osteoblast differentiation [15,37]. A mechanism of HDAC4 repression on Runx 2 has been proposed in which HDAC4 interacts with Runx2 protein to deacetylate Runx2 [38]. Our results indicate the mechanism of HDAC4 acting on Runx2 and MMP-13 in $\mathrm{OA}$ chondrocytes is similar to that found in the growth plate and osteoblasts.

In this study, the reduction of endogenous HDAC4 contributes to the increase of Runx2, type $\mathrm{X}$ collagen, and MMP-13 in OA chondrocytes. We found that the overexpression of HDAC4 decreased the mRNA level of MMP1, MMP3, MMP-13, type X collagen, Ihh ADAMTS-4 and -5 , and increased the mRNA of type II collagen. HDAC4. inhibits chondrocyte hypertrophy by binding to and inhibiting Runx2 and MMP-13 [14,26]. Runx2 is a wellknown transcriptional factor that induces MMP-13, Ihh and ADAMTS-5 production [14,15,39]. Increased Ihh was associated with chondrocyte hypertrophy and upregulation of MMP1, MMP3, and MMP-13 in a mouse OA model and human OA cartilage $[20,40]$. Thus, a mechanism of HDAC4 repression on Runx2 is involved in this phenomenon. Our data also shows that overexpression of HDAC4 not only decreased the expression of IL-1 beta, Cox 2 and iNos and increased the expression of aggrecan, but also partially blocked the effect of IL- 1 beta on expression of catabolic events in human OA chondrocytes. IL-1 beta plays a critical role in the cartilage degeneration [41-43]. However, the mechanism by which HDAC4 regulates IL-1 beta is unclear. Future studies are necessary to investigate the mechanism of HDAC4 action on IL-1 beta. These results suggest that upregulation of HDAC4 may have a chondroprotective effect in patients with earlystage disease.

\section{Conclusions}

In summary, our novel findings indicate, for the first time, that decreased HDAC4 is at least partially responsible for the increase of Runx 2 and OA-related genes in human OA cartilage. This finding will facilitate our understanding of the pathogenesis of degenerative cartilage. Thus, upregulation of HDAC4 may have a chondroprotective effect in OA therapy.

\section{Additional file}

Additional file 1: Figure S1. The transfection efficiency of GFP-HDAC4 or siRNA control. (A) Efficiency of GFP-HDAC4 transfection was $79.1 \%$ (72.6\% to $87.6 \%)$. (B) Efficiency of siRNA control transfection was $88.2 \%$ (85.4\% to $91.2 \%)$. The percentage of cells that are GFP-positive was detected using fluorescence microscopy. Approximately 300 cells from three independent experiments were scored.

\section{Abbreviations}

ANOVA: analysis of variance; DMEM: Dulbecco's modified Eagle's medium; FBS: fetal bovine serum; GEP: green fluorescent protein; HDAC4: histone deacetylase 4; lgG: immunoglobulin G; IL: interleukin; MMPs: matrix metalloproteases; OA: osteoarthritis; PBS: phosphate-buffered saline; RunX2: runt-related transcription factor-2; SD: standard deviation; siRNA: small interfering RNA; TBS: Tris-buffered saline; TSA: trichostatin A.

\section{Competing interests}

The authors declare that they have no competing interests.

\section{Authors' contributions}

KC participated in the design of study, drafted and revised the manuscript. LW conceived of the study, revised the manuscript and performed the statistical analysis. ZQZ prepared the cartilage specimens and help to revise the manuscript. LG carried out the molecular genetic studies and performed the statistical analysis. CMZ carried out the immunoassays and helped to perform the statistical analysis. YPL carried out chondrocyte isolation and primary culture. SWW help to revise the manuscript and performed the statistical analysis. PCL prepared the cartilage specimens and drafted the manuscript. CQS prepared the DNA constructs and carried out the transfections. XJS carried out the reporter gene assays and performed the statistical analysis. XCW conceived of the study, and participated in its design and coordination and helped to draft the manuscript. All authors read and approved the final manuscript.

\section{Acknowledgments}

The project was supported by Grant R01AR059142 from NIH/NIAMS and an Institutional Development Award (IDeA) P20GM104937 from NIH/NIGMS, NSFC 81071495, 81171676 and 31271033, SXNSF 2011011042 , SXISTCP 2013081050 and the Arthritis National Research Foundation (ANRF). The content is solely the responsibility of the authors and does not necessarily represent the official view of the National Institutes of Health. The authors gratefully acknowledge Ericka M. Bueno, Ph.D. for help with the manuscript preparation and editorial services. The authors gratefully acknowledge Dr. Mary B. Goldring for help with the C28/12 human chondrocytes provided for this study. 
Received: 11 March 2014 Accepted: 12 November 2014

Published online: 26 November 2014

\section{References}

1. Hoyland JA, Thomas JT, Donn R, Marriott A, Ayad S, Boot-Handford RP, Grant ME, Freemont AJ: Distribution of type X collagen mRNA in normal and osteoarthritic human cartilage. Bone Miner 1991, 15:151-163.

2. von der Mark K, Kirsch T, Nerlich A, Kuss A, Weseloh G, Glückert K, Stöss H: Type $\mathrm{X}$ collagen synthesis in human osteoarthritic cartilage. Indication of chondrocyte hypertrophy. Arthritis Rheum 1992, 35:806-811.

3. Aigner T, Reichenberger E, Bertling W, Kirsch T, Stoss H, Von der Mark K: Type $X$ collagen expression in osteoarthritic and rheumatoid articular cartilage. Virchows Archiv B Cell Pathol Incl Mol Pathol 1993, 63:205-211.

4. Gouttenoire J, Valcourt U, Ronziere MC, Aubert-Foucher E, Mallein-Gerin F, Herbage D: Modulation of collagen synthesis in normal and osteoarthritic cartilage. Biorheology 2004, 41:535-542.

5. Aigner T, Soder S, Gebhard PM, McAlinden A, Haag J: Mechanisms of disease: role of chondrocytes in the pathogenesis of osteoarthritis-structure, chaos and senescence. Nat Clin Pract Rheumatol 2007, 3:391-399.

6. Slagboom E, Meulenbelt I: Genetics of osteoarthritis: early developmental clues to an old disease. Nat Clin Pract Rheumatol 2008, 4:563.

7. Inada M, Wang Y, Byrne MH, Rahman MU, Miyaura C, López-Otín C, Krane SM: Critical roles for collagenase-3 (Mmp13) in development of growth plate cartilage and in endochondral ossification. Proc Natl Acad Sci U S A 2004, 101:17192-17197.

8. Wang $Y$, Middleton F, Horton JA, Reichel L, Farnum CE, Damron TA: Microarray analysis of proliferative and hypertrophic growth plate zones identifies differentiation markers and signal pathways. Bone 2004, 35:1273-1293.

9. Kamekura S, Hoshi K, Shimoaka T, Chung U, Chikuda H, Yamada T, Uchida M, Ogata N, Seichi A, Nakamura K, Kawaguchi H: Osteoarthritis development in novel experimental mouse models induced by knee joint instability. Osteoarthritis Cartilage 2005, 13:632-641.

10. Kamekura S, Kawasaki Y, Hoshi K, Shimoaka T, Chikuda H, Maruyama Z, Komori T, Sato S, Takeda S, Karsenty G, Nakamura K, Chung UI, Kawaguchi H: Contribution of runt-related transcription factor 2 to the pathogenesis of osteoarthritis in mice after induction of knee joint instability. Arthritis Rheum 2006, 54:2462-2470.

11. Pacifici M, Koyama E, Iwamoto M: Mechanisms of synovial joint and articular cartilage formation: recent advances, but many lingering mysteries. Birth Defects Res C Embryo Today 2005, 75:237-248.

12. Kirsch T, Swoboda B, Nah H: Activation of annexin II and V expression terminal differentiation, mineralization and apoptosis in human osteoarthritic cartilage. Osteoarthritis Cartilage 2000, 8:294-302.

13. Pfander D, Swoboda B, Kirsch T: Expression of early and late differentiation markers (proliferating cell nuclear antigen, syndecan-3, annexin $\mathrm{VI}$, and alkaline phosphatase) by human osteoarthritic chondrocytes. Am J Pathol 2001, 159:1777-1783.

14. Vega RB, Matsuda K, Oh J, Barbosa AC, Yang X, Meadows E, McAnally J, Pomajzl C, Shelton JM, Richardson JA, Karsenty G, Olson EN: Histone deacetylase 4 controls chondrocyte hypertrophy during skeletogenesis. Cell 2004, 119:555-566.

15. Shimizu E, Selvamurugan N, Westendorf JJ, Olson EN, Partridge NC: HDAC4 represses matrix metalloproteinase-13 transcription in osteoblastic cells, and parathyroid hormone controls this repression. J Biol Chem 2010, 285:9616-9626.

16. Wang X, Manner PA, Horner A, Shum L, Tuan RS, Nuckolls GH: Regulation of MMP-13 expression by RUNX2 and FGF2 in osteoarthritic cartilage. Osteoarthritis Cartilage 2004, 12:963-973.

17. Higashikawa A, Saito T, Ikeda T, Kamekura S, Kawamura N, Kan A, Oshima Y, Ohba S, Ogata N, Takeshita K, Nakamura K, Chung UI, Kawaguchi H: Identification of the core element responsive to runt-related transcription factor 2 in the promoter of human type $X$ collagen gene. Arthritis Rheum 2009, 60:166-178.

18. Higashiyama R, Miyaki S, Yamashita S, Yoshitaka T, Lindman G, Ito Y, Sasho T, Takahashi K, Lotz M, Asahara H: Correlation between MMP-13 and HDAC7 expression in human knee osteoarthritis. Mod Rheumatol 2010, 20:11-17.

19. Carlson CS, Loeser RF, Purser CB, Gardin JF, Jerome CP: Osteoarthritis in cynomolgus macaques. III: effects of age, gender, and subchondral bone thickness on the severity of disease. J Bone Min Res 1996, 11:1209-1217.
20. Wei F, Zhou J, Wei X, Zhang J, Fleming BC, Terek R, Pei M, Chen Q, Liu T, Wei L: Activation of Indian hedgehog promotes chondrocyte hypertrophy and upregulation of MMP-13 in human osteoarthritic cartilage. Osteoarthritis Cartilage 2012, 20:755-763.

21. Chatila T, Anderson KA, Ho N, Means AR: A unique phosphorylation-dependent mechanism for the activation of $\mathrm{Ca} 2+/$ calmodulin-dependent protein kinase type IV/GR. J Biol Chem 1996, 271:21542-21548.

22. Drissi H, LuC Q, Shakoori R, Chuva De Sousa Lopes S, Choi JY, Terry A, Hu M, Jones S, Neil JC, Lian JB: Transcriptional autoregulation of the bone related CBFA1/RUNX2 gene. J Cell Physiol 2000, 184:341-350.

23. Im HJ, Pacione C, Chubinskaya S, Van Wijnen AJ, Sun Y, Loeser RF: Inhibitory effects of insulin-like growth factor-1 and osteogenic protein-1 on fibronectin fragment- and interleukin-1 beta-stimulated matrix metalloproteinase-13 expression in human chondrocytes. $\mathrm{J}$ Biol Chem 2003, 278:25386-25394.

24. Livak KJ, Schmittgen TD: Analysis of relative gene expression data using real-time quantitative PCR and the 2(-Delta Delta $C(T))$. Methods 2001, 25:402-408

25. Claassen $H$, Schicht M, Brandt J, Reuse $K$, Schädlich R, Goldring MB, Guddat SS, Thate A, Paulsen F: C-28/12 and T/C-28a2 chondrocytes as well as human primary articular chondrocytes express sex hormone and insulin receptors-useful cells in study of cartilage metabolism. Ann Anat 2011, 20:23-29.

26. Guan $Y$, Chen $Q$, Yang X, Haines P, Pei M, Terek R, Wei X, Zhao T, Wei L: Subcellular relocation of histone deacetylase 4 regulates growth plate chondrocyte differentiation through $\mathrm{Ca} 2+$ /calmodulin-dependent kinase IV. Am J Physiol Cell Physiol 2012, 303:33-40.

27. Fischle W, Emiliani S, Hendzel MJ, Nagase T, Nomura N, Voelter W, Verdin E: A new family of human histone deacetylases related to Saccharomyces cerevisiae HDA1p. J Biol Chem 1999, 274:11713-11720

28. Fischle $W$, Kiermer $\mathrm{V}$, Dequiedt $F$, Verdin $\mathrm{E}$ : The emerging role of class II histone deacetylases. Biochem Cell Biol 2001, 79:337-348.

29. Aigner T, Dietz U, Stoss H, von der Mark K: Differential expression of collagen types I, II, III, and X in human osteophytes. Lab Invest 1995, 73:236-243

30. Bau B, Gebhard PM, Haag J, Knorr T, Bartnik E, Aigner T: Relative messenger RNA expression profiling of collagenases and aggrecanases in human articular chondrocytes in vivo and in vitro. Arthritis Rheum 2002, 46:2648-2657.

31. Clements DN, Carter SD, Innes JF, Ollier WER, Day PJR: Analysis of normal and osteoarthritic canine cartilage mRNA expression by quantitative polymerase chain reaction. Arthritis Res Ther 2006, 8:158.

32. Little CB, Barai A, Burkhardt D, Smith SM, Fosang AJ, Werb Z, Shah M, Thompson EW: Matrix metalloproteinase 13-deficient mice are resistant to osteoarthritic cartilage erosion but not chondrocyte hypertrophy or osteophyte development. Arthritis Rheum 2009, 60:3723-3733.

33. Kawaguchi $\mathrm{H}$ : Endochondral ossification signals in cartilage degradation during osteoarthritis progression in experimental mouse models. Mol Cells 2008, 25:1-6.

34. Billinghurst RC, Dahlberg L, lonescu M, Reiner A, Bourne R, Rorabeck C, Mitchell P, Hambor J, Diekmann O, Tschesche H, Chen J, Van Wart H, Poole AR: Enhanced cleavage of type II collagen by collagenases in osteoarthritic articular cartilage. J Clin Invest 1997, 99:1534-1545.

35. Poole AR, Nelson F, Dahlberg L, Tchetina E, Kobayashi M, Yasuda T, Laverty S, Squires G, Kojima T, Wu W, Billinghurst RC: Proteolysis of the collagen fibril in osteoarthritis. Biochem Soc Symp 2003, 70:115-123.

36. Tardif G, Reboul P, Pelletier JP, Martel-Pelletier J: Ten years in the life of an enzyme: the story of the human MMP-13 (collagenase-3). Mod Rheumatol 2004, 14:197-204.

37. Kang JS, Alliston T, Delston R, Derynck R: Repression of Runx2 function by TGF-beta through recruitment of class II histone deacetylases by Smad3. EMBO J 2005, 24:2543-2555.

38. Jeon EJ, Lee KY, Choi NS, Lee MH, Kim HN, Jin YH, Ryoo HM, Choi JY, Yoshida M, Nishino N, Oh BC, Lee KS, Lee YH, Bae SC: Bone morphogenetic protein-2 stimulates Runx2 acetylation. J Biol Chem 2006 281:16502-16511.

39. Thirunavukkarasu K, Pei Y, Wei T: Characterization of the human ADAMTS-5 (aggrecanase-2) gene promoter. Mol Biol Rep 2007, 34:225-231.

40. Lin AC, Seeto BL, Bartoszko JM, Khoury MA, Whetstone H, Ho L, Hsu C, Ali SA, Alman BA: Modulating hedgehog signaling can attenuate the severity of osteoarthritis. Nat Med 2009, 15:1421-1425. 
41. Kapoor M, Martel-Pelletier J, Lajeunesse D, Pelletier JP, Fahmi H: Role of roinflammatory cytokines in the pathophysiology of osteoarthritis. Nat Rev Rheumatol 2011, 7:33-42.

42. Daheshia M, Yao JQ: The interleukin 1 beta pathway in the pathogenesis of osteoarthritis. J Rheumatol 2008, 35:2306-2312.

43. Kobayashi M, Squires GR, Mousa A, Tanzer M, Zukor DJ, Antoniou J, Feige U, Poole AR: Role of interleukin-1 and tumor necrosis factor alpha in matrix degradation of human osteoarthritic cartilage. Arthritis Rheum 2005, 52:128-135.

doi:10.1186/s13075-014-0491-3

Cite this article as: Cao et al.: Decreased histone deacetylase 4 is associated with human osteoarthritis cartilage degeneration by releasing histone deacetylase 4 inhibition of runt-related transcription factor- 2 and increasing osteoarthritis-related genes: a novel mechanism of human osteoarthritis cartilage degeneration. Arthritis Research \& Therapy 2014 16:491.

\section{Submit your next manuscript to BioMed Central and take full advantage of:}

- Convenient online submission

- Thorough peer review

- No space constraints or color figure charges

- Immediate publication on acceptance

- Inclusion in PubMed, CAS, Scopus and Google Scholar

- Research which is freely available for redistribution 